\title{
Rheumatoid and psoriatic knee synovitis: clinical, grey scale, and power Doppler ultrasound assessment of the response to etanercept
}

\author{
U Fiocco, F Ferro, M Vezzù, L Cozzi, C Checchetto, P Sfriso, C Botsios, L Ciprian, \\ G Armellin, R Nardacchione, A Piccoli, S Todesco, L Rubaltelli
}

Ann Rheum Dis 2005;64:899-905. doi: 10.1136/ard.2004.025585

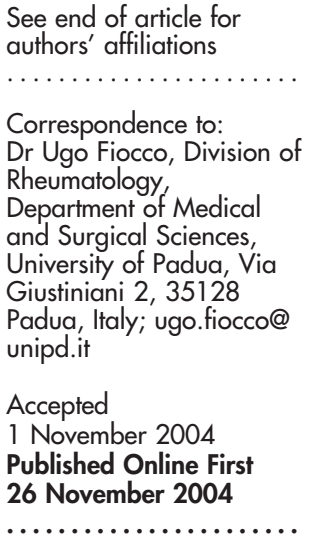

\begin{abstract}
Objective: To determine the effect of tumour necrosis factor $\alpha$ (TNF $\alpha$ ) blockade with etanercept in refractory knee joint synovitis (KJS) in rheumatoid and psoriatic arthritis, by local and systemic disease activity assessment and combined grey scale and power Doppler ultrasonographic monitoring.

Methods: 27 knees affected by rheumatoid KJS $(n=12)$ and psoriatic KJS $(n=8)$ were assessed before receiving treatment and at 3 and 12 months' follow up. Time dependent clinical changes in disease activity were monitored by $C$ reactive protein, erythrocyte sedimentation rate (ESR), global health status (GHS), and Ritchie (RAl) and knee joint articular (KJAl) indices; synovial changes were monitored by ultrasonographic and power Doppler indices for grey scale synovial thickening and for distinct intrasynovial vessel power Doppler flow configurations (fluid/synovium interface (F/SI-PD) and pannus/ cartilage interface (P/CI-PD)). Interobserver and intraobserver variability of grey scale and power Doppler ultrasonographic was evaluated. Response to treatment was assessed by analysis of variance for repeated measures on clinical and ultrasonographic variables.

Results: Rapid ( 3 months) reduction in F/SI-PD flow ( $p<0.001$ ), parallel to reductions of $C$ reactive protein $(p<0.05)$, ESR $(p<0.001), \mathrm{KJAl}(p<0.002)$, RAl, and GHS ( $p<0.001)$, was sustained at 12 months when it was accompanied by reduction in both synovial thickening and P/CI-PD flow $(p<0.001)$. No differences (ANOVA) were noted at baseline or at 12 months in clinical and ultrasonographic variables between either the rheumatoid or the psoriatic KJS groups.

Conclusion: Grey scale and power Doppler ultrasonography are reliable measures of long term change in rheumatoid and psoriatic KJS disease activity in response to anti-TNF $\alpha$ treatment with etanercept.
\end{abstract}

R efractory rheumatoid knee joint synovitis (KJS) is defined as the persistence of active synovitis after at least six months of aggressive local and systemic medical management. ${ }^{1}$ Relapse of KJS is common and may occur at any time during treatment with traditional systemic disease modifying antirheumatic drugs (DMARDs). The negative impact in the long term outcome of refractory KJS, which clearly emerges from the recently reported incidence of knee joint arthroplasty-representing 68\% of all disease related surgical procedures and $57 \%$ of all total joint replacements in large series of rheumatoid patients ${ }^{2}-$ highlights the need for new approaches to the treatment of KJS. Polyarticular psoriatic arthritis results in joint damage, disability, and increased mortality, showing a comparable level of functional and radiological disease progression to rheumatoid arthritis. ${ }^{34}$ Actively inflamed joints resistant to standard DMARD treatment are common in refractory psoriatic disease $^{56}$ and recent analysis of DMARD treatment for psoriatic arthritis suggests that both lack of efficacy and adverse effects result in high rates of discontinuation. ${ }^{7}$

Among the new biological agents, one inhibitor of tumour necrosis factor $\alpha(\mathrm{TNF} \alpha)$, etanercept-a fully human recombinant (p75) TNF receptor-FC dimeric fusion protein (TNFR:FC)-is the first biological response modifier approved in the USA for use in rheumatoid patients with moderately to severely active arthritis, regardless of previous DMARD treatment. It has expanded indications for inhibiting the progression of structural damage. At present, etanercept is also the only approved treatment for both inhibition of structural damage and reduction of signs and symptoms in patients with psoriatic arthritis. ${ }^{8}$
However, whether the strategy of TNF $\alpha$ blockade by soluble TNFR:Fc can relieve the orthopaedic surgical burden in rheumatoid and psoriatic KJS by retarding pannus induced articular destruction remains unknown.

New developments in imaging in rheumatoid arthritis by recent technical advances in high frequency and power Doppler ultrasound and magnetic resonance imaging (MRI) ${ }^{9}$ can provide essential information for new management strategies. ${ }^{10}$ Conventional ultrasonographic imaging of synovial proliferation has been shown to correlate with both arthroscopic $^{11}$ and articular indices of KJS, and to be useful objective method for monitoring response to therapy. ${ }^{12}$ Power Doppler ultrasonography-which has been shown to increase the specificity of traditional grey scale sonography, ${ }^{13}$ allowing improved detection and quantification of inflammation ${ }^{14}$ has been used to differentiate between hypervascular and fibrous pannus ${ }^{15}$ and to assess the response to treatment of joint synovitis. ${ }^{16} 17$

Until now, no study has determined the effect of TNF $\alpha$ blockade in refractory rheumatoid and psoriatic knee synovitis by combining grey scale and power Doppler ultrasonographic monitoring. Our aim in this study was to

Abbreviations: DMARD, disease modifying antirheumatic drug; $\mathrm{F} / \mathrm{SI}-$ $P D$, fluid/synovium interface power Doppler flow configuration; GHS, global health status; KJAI, knee joint articular index; KJS, knee joint synovitis; LPPR, lateral parapatellar recess; MPPR, medial parapatellar recess; P/CI-PD, pannus/cartilage interface power Doppler flow configuration; RAl, Ritchie articular index; SPR, suprapatellar recess; $\mathrm{TNF} \alpha$, tumour necrosis factor $\alpha$; TNFR:Fc, tumour necrosis factor receptor-Fc dimeric fusion protein 
assess time dependent changes in disease activity indices and in synovial thickening and intrasynovial power Doppler vessel flow ultrasonography in refractory rheumatoid and psoriatic KJS, as a measure of the therapeutic response to etanercept.

\section{METHODS}

\section{Patients}

The study was designed as an open label, single centre, 12 months prospective study to assess the clinical and sonographic KJS response to TNFR:Fc. The protocol was approved by the local human research committee. All patients gave their written informed consent before entering the study.

We enrolled 20 consecutive patients ( 27 knees) affected by refractory KJS attending the rheumatology clinic at Padua General Hospital.

All knees showed clinical evidence of joint effusion (positive bulge sign or ballottement of the patella, or both); 12 patients had rheumatoid arthritis (17 knees) and eight had polyarticular psoriatic arthritis ( 10 knees), as defined by generally accepted criteria. ${ }^{18}{ }^{19}$ In view of the difficulties in distinguishing the polyarthritic form of psoriatic arthritis from rheumatoid arthritis, patients with psoriatic arthritis were examined for nail lesions (pits and onycholysis), psoriasis or family history of psoriasis, dactylitis, and distal interphalangeal joint involvement occurring since the onset of disease, along with rheumatoid factor negativity or low titre ( $\leqslant 40 \mathrm{KU} /$ litre) and radiographic/MRI evidence of axial involvement, and entheseal associated pathology. ${ }^{21}$ No patient had psoriasis affecting more than 3\% of the body surface area on entering the study.

All patients suffered from persistent active synovitis of the knee (characterised by pain, tenderness, and effusion), which had proved resistant to intra-articular corticosteroid injections (at least six weeks before entry into the study) and to at least 12 months of second line treatment. On entry all patients were non-responders to methotrexate monotherapy ( $\geqslant 15 \mathrm{mg} /$ week); methotrexate in combination with another DMARD, at standard target doses or, when there was intolerance of methotrexate, to two different DMARDs in combination. The baseline clinical and demographic characteristics of the cohort are shown in tables 1 and 2. The rheumatoid and psoriatic KJS groups had received on average 3.3 and 3.8 intra-articular steroid injections to the knee (triamcinolone acetonide $40 \mathrm{mg}$ ), respectively, in the previous six months, up to one month before study entry (table 2).

Exclusion criteria included pregnancy and known significant concurrent medical disease.

On study entry, patients were treated with $25 \mathrm{mg}$ subcutaneous etanercept twice a week for 12 months. Previous DMARDs (methotrexate, sulfasalazine, hydroxychloroquine, ciclosporine), previous DMARD combinations, as well as low dose oral corticosteroids ( $\leqslant 10 \mathrm{mg}$ prednisone) with or without non-steroid anti-inflammatory agents, were continued in stable appropriate doses. Patients stopping previous DMARDs owing to adverse effects during the screening period of the study were allowed to continue with etanercept monotherapy (tables 1 and 2). Use of intraarticular corticosteroids was not allowed. Concomitant treatment was monitored and recorded throughout the study.

Single blind clinical and laboratory assessments of disease activity markers were made at enrolment (T0) and at 3 and 12 months (T12), and consisted of physical examination, vital signs, measures of disease activity, concomitant drug treatment, combined grey scale and power Doppler ultrasonographic assessment, and monitoring of any adverse events.

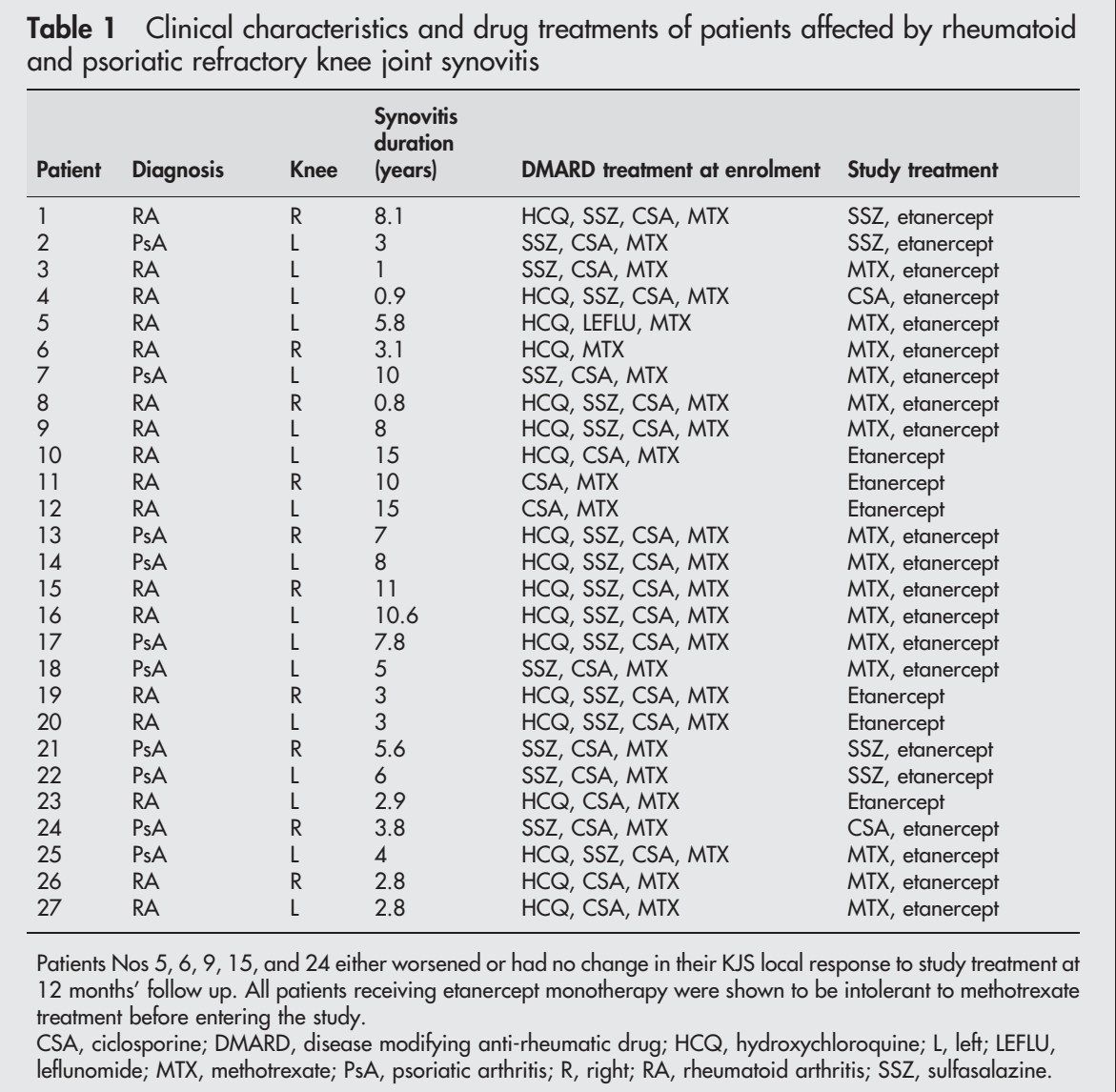


Table 2 Baseline clinical characteristics of patients affected by rheumatoid and psoriatic refractory knee joint synovitis

\begin{tabular}{lll} 
& RA & PsA \\
\hline Age (years) (mean (SD)) & $50.6(17.3)$ & $43.1(14.9)$ \\
Female (n (\%)) & $11(91.6)$ & $5(62.5)$ \\
Polyarticular joint involvement (n (\%)) & $12(100)$ & $8(100)$ \\
RF positivity (n (\%)) & $7(58)$ & $0(0)$ \\
Entheseal involvement (n (\%)) & $0(0)$ & $7(87.5)$ \\
Axial involvement (n (\%)) & $0(0)$ & $6(75)$ \\
Knee joint involvement (n) & 17 & 10 \\
KJS duration (years) (mean (SD)) & $6.1(4.8)$ & $6.0(2.2)$ \\
ESR (mm/h) (mean (SD)) & $50.1(25.1)$ & $41.5(28.2)$ \\
C reactive protein (mg/dl) (mean (SD)) & $33.9(21.9)$ & $22.6(11.2)$ \\
Knee joint articular index (mean (SD)) & $3.9(2.4)$ & $4.3(2.7)$ \\
Mean DMARD use (n) & 3.3 & 3.4 \\
Mean IA knee joint injections (n) & 3.3 & 3.8 \\
Methotrexate use (n (\%)) & $9(52.9)$ & $6(60)$ \\
Etanercept monotherapy (n (\%)) & $6(35.3)$ & $0(0)$ \\
Etanercept combination therapy (n (\%)) & $11(64.7)$ & $10(100)$ \\
Prednisone (n (\%)) & $17(100)$ & $10(100)$ \\
\hline DMARD, disease modifying antirheumatic drug; ESR, erythrocyte \\
sedimentation rate; IA, intra-articular; KJS, knee joint synovitis; PsA, \\
psoriatic arthritis; RA, rheumatoid arthritis; RF, rheumatoid factor.
\end{tabular}

Safety assessment included monthly repetition of laboratory tests (urinalysis, complete blood cell count, renal and liver function tests).

Measures of psoriatic and rheumatoid KJS disease activity included:

- knee joint articular index (KJAI): sum of scores of tenderness (0-3), joint swelling (0-3), ballottement of patella or "bulge sign" (0-2), and range of knee joint flexion (0-3) and extension (0-3) (KJAI, range 0-14) ${ }^{22}$;3;

- Ritchie articular index (RAI): assessment of 78 joints for tenderness (0-3) and 76 for swelling (0-3);

- global health status (GHS, range 0-100);

- serum concentration of $\mathrm{C}$ reactive protein;

- erythrocyte sedimentation rate (ESR).

\section{Ultrasound evaluation}

Grey scale assessment

All examinations were carried out using a high frequency linear transducer (10 MHz Elegra, Siemens, Erlangen, Germany) by the same two experienced observers, who were not aware of the clinical findings in the patients.

Standardised anatomical guidelines of the scans in the three recesses of the knee-suprapatellar recess (SPR) and lateral and medial parapatellar recesses (LPPR, MPPR)were used, as previously described. ${ }^{11}$
The synovial thickness of the SPR was determined by scanning the zone between the prefemoral (posterior suprapatellar) fat pad and the upper margin of the femoral cartilage (supine position; knee joint extended; bicipes femoris at rest). At the level of the MPPR and LPPR, the vertical edge along the medial and lateral margins of the knee cap (bicipes femoris contracted) was identified by scanning. Nodular vegetations, when present, were measured in their entire thickness. On entry to the study, each knee was evaluated as a whole, and the worst area of thickening detected between the three recesses was measured; the resulting value was assumed to be a measure of synovial thickness. $^{12}$

\section{Power Doppler assessment}

Power Doppler sonography was set for high sensitivity, with a low wall filter to allow detection of vessels with low blood flow. Pulse repetition frequency was $750-1200 \mathrm{~Hz}$ and medium persistence was used. The colour gain was increased until background noise appeared and then reduced until noise was suppressed, thus ensuring maximum sensitivity.

A combined grey scale and power Doppler study was carried out in the three distinct joint recesses, to assess power Doppler flow signals in two orthogonal planes in the pannus areas (more than $3 \mathrm{~mm}$ of synovial thickness). ${ }^{24}$

For each ultrasonographic scan, the power Doppler signal of the synovial membrane was graded on a $0-3$ scale $(0=$ normal, undetectable power Doppler vessel signals in ultrasonographic synovial thickening area; $1=$ mild hyperaemia; 2 = moderate hyperaemia; 3 = marked hyperaemia), if intrasynovial power Doppler flow signal distribution was detectable over $<25 \%$, $\leqslant 50 \%$, or $>50 \%$ of the synovial thickening area. The intrasynovial power Doppler flow signals of identifiable vessels were scored (0-3) for distinct spatial arrangements in relation to the fluid/synovium interface (F/SI-PD) and the pannus/cartilage or pannus/ capsule interface (P/CI-PD), according to arthroscopic vascular architecture between synovial villous surfaces and deep pannus layers adhering to cartilage/capsule, as previously described. ${ }^{24}$ Spectral Doppler tracing was used to confirm the presence of true synovial vessel flow for distinct colour Doppler signal spatial arrangements. During the last month before entering the study, two repeat power Doppler ultrasonographic KJS assessments were carried out in 12 knees to determine the intraobserver variation coefficient.

\section{Statistics}

Statistical analysis was done with SPSS software (version 11.5). The effect of treatment over time, classified by KJS disease (rheumatoid arthritis and psoriatic arthritis), was evaluated by analysis of variance for repeated measures using

Table 3 Mean changes in clinical and ultrasound variables of rheumatoid and psoriatic refractory knee joint synovitis over the 12 month follow up period

\begin{tabular}{|c|c|c|c|c|c|}
\hline & Baseline & 3 Months & 12 Months & $\mathbf{F}$ & p Value \\
\hline RAI & $25.72(3.00)$ & $8.23(1.64)^{* \star *}$ & $3.52(1.03)^{\star * \star}$ & 41.2 & $<0.001$ \\
\hline GHS & $40.69(4.31)$ & $64.08(3.47)^{\star * *}$ & $73.28(4.14)^{\star * *}$ & 20.5 & $\leqslant 0.001$ \\
\hline CRP & $28.13(4.84)$ & $15.47(3.75)^{*}$ & $10.60(3.99)^{* *}$ & 5.9 & $<0.005$ \\
\hline ESR & 44.60 (6.91) & $26.58(5.63)^{\star \star *}$ & $21.36(5.15)^{\star \star *}$ & 11.1 & $<0.001$ \\
\hline ST & $6.6(0.6)$ & $6.4(0.6)$ & $4.8(0.5)^{*}$ & 7.6 & $<0.001$ \\
\hline F/SI-PD & $1.31(0.30)$ & $0.63(0.21)^{* * *}$ & $0.44(0.20)^{*}$ & 7.4 & $<0.002$ \\
\hline $\mathrm{P} / \mathrm{Cl}-\mathrm{PD}$ & $1.59(0.21)$ & $1.62(0.18)$ & $0.89(0.18)^{*}$ & 9.6 & $\leqslant 0.001$ \\
\hline KJAI & $3.97(0.64)$ & $2.13(0.41)$ ** & $1.65(0.35)^{\star *}$ & 11.4 & $<0.001$ \\
\hline \multicolumn{6}{|c|}{$\begin{array}{l}\text { Values are mean }(S E M) \text {. } \\
{ }^{*} p \leqslant 0.05 ;{ }^{* *} p \leqslant 0.002 ;{ }^{* * *} p \leqslant 0.001, v \text { baseline. } \\
C R P, C \text { reactive protein; ESR, erythrocyte sedimentation rate; } F / S I-P D \text {, fluid/synovium interface power Doppler } \\
\text { flow configuration; GHS, global health status; } \mathrm{KJAl} \text {, knee joint articular index; } \mathrm{P} / \mathrm{Cl}-\mathrm{PD} \text {, pannus/cartilage interface } \\
\text { power Doppler flow configuration; RAI, Ritchie articular index; } \mathrm{ST} \text {, synovial thickness. }\end{array}$} \\
\hline
\end{tabular}



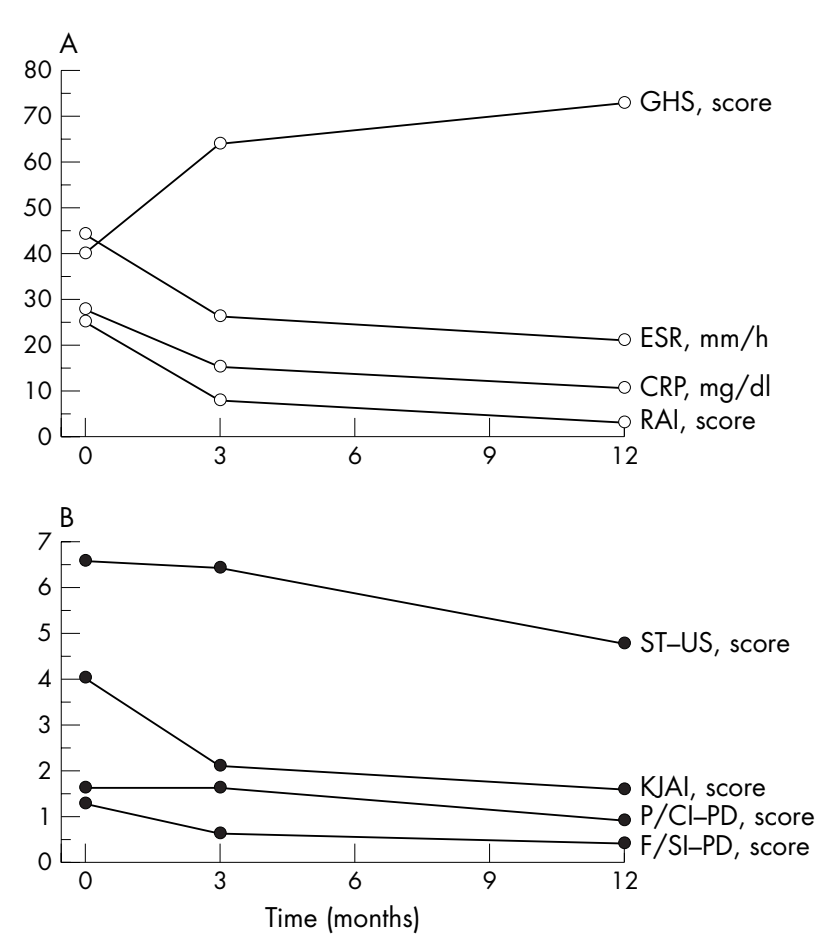

Figure 1 Mean changes in disease activity and ultrasonographic variables over time in rheumatoid and psoriatic refractory knee joint synovitis (KJS). (A) systemic disease activity measurements. (B) local disease activity and ultrasonographic measurements. CRP, $C$ reactive protein; ESR, erythrocyte sedimentation rate; F/SI-PD, fluid/synovium interface power Doppler flow configuration; GHS, global health status; $\mathrm{KJAl}$, knee joint articular index; $\mathrm{P} / \mathrm{Cl}-\mathrm{PD}$, pannus/cartilage interface power Doppler flow configuration; RAI, Ritchie articular index; ST-US, synovial thickness measured by ultrasound.

clinical and sonographic indicators as dependent variables. Means (SEM) were used for data presentation. Interobserver reliability between results of the semiquantitative scoring of the maximum power Doppler flow signal area of enhancement, ${ }^{16}$ obtained by review of power Doppler images by two experienced observers, was measured and the $\kappa$ value (Bonferroni) for interobserver reliability was calculated $(\kappa=-1.0$ represents no agreement, $\kappa=1.0$, perfect agreement). For within observer measurement error, 12 KJS (six rheumatoid, six psoriatic) were studied on two occasions by the same observer within a three week period during the last month before entering the study. Technical errors of measurement of synovial thickening and power Doppler

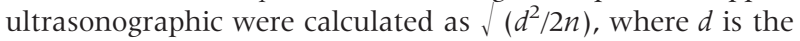
difference between two observations and $n$ is the number of pairs of observations, ${ }^{25}$ and expressed as coefficient of variation $(\mathrm{CV})$ - that is, the percentage of technical error divided by the overall mean of the measurements.

\section{RESULTS}

The patient characteristics are given in tables 1 and 2 . Etanercept treatment was well tolerated by all patients, with no major adverse events and in particular no severe infections. Three patients (all with rheumatoid arthritis) had to discontinue treatment for two consecutive injections after the first three months of follow up because of minor infections (erysipelas, paronychia of the second finger of the right hand, and labial Herpes simplex).

Mean changes in clinical and ultrasonographic variables over time are shown in table 3 and fig 1. After treatment, all variables changed significantly over the 12 month follow up period: all indices of disease activity decreased after three months, other than GHS, which increased significantly. Among grey scale and power Doppler ultrasonographic findings, only F/SI-PD flow decreased at three months.

At the end of the follow up all patients had marked and significant improvement in all systemic measures of rheumatoid and psoriatic KJS disease activity.

Clinical and ultrasound data on changes in clinical and ultrasonographic variables over time in the rheumatoid and psoriatic KJS groups, and a comparison of the response at 12 months between the two groups, are given in table 4 . No differences were found in any of the clinical and ultrasound variables between the rheumatoid and the psoriatic arthritis groups, as indicated by the comparable mean values between baseline and 12 months (table 4 ).

In 22 knees $(81 \%)$ the KJAI score improved by more than $50 \%$ at the 12 month follow up. In five knees, KJS local disease activity worsened or remained unchanged (nonresponder group), as follows: no change in three knees (11\%; two with rheumatoid arthritis and one with psoriatic arthritis; patients 9, 15, and 24, respectively); and the KJAI score worsened in two knees (7\%; both with rheumatoid arthritis; patients 5 and 6).

The baseline clinical characteristics (KJS duration), mean DMARD use, measures of local and systemic disease activity, synovial grey scale ultrasound, and power Doppler ultrasonographic measures were similar between the non-responder group and the rheumatoid and psoriatic KJS groups. The non-responder group had a higher baseline RAI than the responder groups (non-responder group, 37.2; responder groups: rheumatoid KJS, 30.6; psoriatic KJS, 17.1).

No patient underwent either arthroscopic synovectomy or knee joint arthroplasty during the follow up period.

The $\kappa$ value, a chance corrected measure of agreement between pairs of observers, was $1(100 \%)$ for both synovial thickness and power Doppler ultrasonographic grading.

Within-observer coefficients of variation were $11 \%$ for synovial thickness, $14 \%$ for P/CI-PD, and $18 \%$ for F/SI-PD ultrasonographic flow, respectively.

\section{DISCUSSION}

The aim of our longitudinal study was to determine the effect of TNF $\alpha$ blockade with etanercept in rheumatoid and psoriatic KJS, by assessing the treatment induced time dependent changes in disease activity and in combined grey scale and power Doppler ultrasonographic outcome measures.

The initial three months of etanercept treatment were associated with a definite reduction in both local and systemic disease activity, as detected by subjective and objective clinical end points and biochemical markers (table 2). Our findings, in agreement with previous data, support the view that TNF $\alpha$ blockade is effective at rapidly suppressing synovial inflammation, ${ }^{26-28}$ even in patients with long lasting rheumatoid and psoriatic KJS, who have an inadequate response to DMARD combinations and local steroid treatment. ${ }^{28}{ }^{29}$ Nevertheless, our data are preliminary because of the small numbers of patients examined (low statistical power) and uncontrolled nature of the study. Thus the potential clinical relevance has to be proven in further larger studies.

The changes in clinical and ultrasonographic findings were concordant in showing a sustained reduction in disease activity indices, particularly at 12 months, at which time the reduction in ultrasonographic synovial thickening appeared highly significant (table 3 ). This finding is noteworthy in severe resistant psoriatic KJS, as the level of inflammation is an important predictor of disease progression, and most joint damage appears to occur early in the course of psoriatic arthritis. $^{30}$ 
Table 4 Comparison of responses of the clinical and ultrasound variables at the 12 month follow up between the rheumatoid and the psoriatic knee joint synovitis groups

\begin{tabular}{|c|c|c|c|c|c|}
\hline \multirow[b]{2}{*}{ Variable } & \multirow[b]{2}{*}{ KJS } & \multirow[b]{2}{*}{ Baseline } & \multirow[b]{2}{*}{12 months } & \multicolumn{2}{|c|}{ ANOVA by diagnosis } \\
\hline & & & & $\mathbf{F}$ & $\mathrm{p}$ Value \\
\hline RAI & $\begin{array}{l}\text { RA } \\
\text { PsA }\end{array}$ & $\begin{array}{l}30.6(3.1) \\
17.1(3.8)\end{array}$ & $\begin{array}{l}4.4(1.1) \\
3.1(1.3)\end{array}$ & 4.3 & NS \\
\hline \multirow[b]{2}{*}{ GHS } & RA & $39.5(4.3)$ & $75.3(4.8)$ & 2.6 & \multirow[b]{2}{*}{ NS } \\
\hline & PsA & $43.8(5.3)$ & $63.3(5.9)$ & & \\
\hline \multirow{2}{*}{ CRP } & RA & $34.2(5.1)$ & $8.9(4.3)$ & 1.3 & \multirow{2}{*}{ NS } \\
\hline & PsA & $22.6(6.2)$ & $19.6(5.2)$ & & \\
\hline \multirow{2}{*}{ ESR } & RA & $51.4(7.0)$ & $20.2(5.7)$ & 0.7 & \multirow{2}{*}{ NS } \\
\hline & PsA & $41.5(8.6)$ & $34.3(7.0)$ & & \\
\hline \multirow{2}{*}{ ST } & RA & $6.0(0.6)$ & $5.5(0.6)$ & 0.1 & \multirow{2}{*}{ NS } \\
\hline & PsA & $7.2(0.8)$ & $4.4(0.7)$ & & \\
\hline \multirow{2}{*}{ F/SI-PD } & RA & $1.7(0.3)$ & $0.6(0.2)$ & 0.01 & \multirow[b]{2}{*}{ NS } \\
\hline & PsA & $1.3(0.4)$ & $0.5(0.2)$ & & \\
\hline \multirow{2}{*}{ P/CI-PD } & RA & $1.8(0.2)$ & $1.7(0.3)$ & 0.1 & \multirow{2}{*}{ NS } \\
\hline & PsA & $1.1(0.2)$ & $0.9(0.2)$ & & \\
\hline \multirow[b]{2}{*}{ KJAI } & RA & $4.1(0.6)$ & $1.5(0.4)$ & 1.2 & \multirow{2}{*}{ NS } \\
\hline & Ps $\mathrm{A}$ & $4.3(0.8)$ & $2.0(0.4)$ & & \\
\hline
\end{tabular}

To the best of our knowledge, there are no reports of sustained regression of ultrasonographic synovial thickening after TNF $\alpha$ blockade in rheumatoid or psoriatic knee joints resistant to DMARD treatment. The ultrasonographic pannus response in the hand joints after infliximab treatment was examined in two recent studies, ${ }^{31} 32$ reporting a decrease in grey scale synovial ultrasonographic thickness in small hand joint rheumatoid synovitis at the early times of six and 18 weeks. Interestingly, baseline synovial vascularity was related both to a lower degree of synovial response to infliximab ${ }^{31}$ and to later radiological changes. ${ }^{32}$

Our findings in large joints with highly vascular and proliferative synovitis ${ }^{23}$ provide objective evidence of a sustained therapeutic effect of TNF $\alpha$ blockade with etanercept on the process of pannus formation in rheumatoid and psoriatic arthritis.

Previously, long term monitoring of the response to arthroscopic synovectomy combined with DMARD monotherapy in rheumatoid and psoriatic KJS, using either contrast enhanced MRI (CE-MRI) assessment ${ }^{33}$ or grey scale ultrasonographic evaluation, ${ }^{12}$ provided no evidence of pannus regression, in spite of an immediate post-synovectomy effect. Etanercept treatment already induced a sustained ( six months) reduction in knee and hip synovitis, shown by CE-MRI, in patients with resistant spondylarthropathy. ${ }^{34}$

Looking at time dependent synovial power Doppler flow changes, reduction of F/SI (that is, power Doppler flow in superficial pannus layer and synovial villi) was found to precede changes in ultrasonographic synovial thickening, appearing earlier compared with P/CI (that is, power Doppler flow in the deep pannus layer), and matching changes in disease activity indices (fig 1).

Synovial villous power Doppler flow variations fitted preliminary observations of rapid synovial power Doppler flow changes in rheumatoid hand joints-matching changes in disease activity after etanercept treatment ${ }^{35}{ }^{36}$ and the finding of an early reduction (85\%) in the uptake of gadolinium-diethylenetriaminepentaacetic acid (DTPA) shown by dynamic MRI and detected in peripheral joints of patients with psoriatic arthritis following TNF $\alpha$ blockade by infliximab. ${ }^{27}$ CE-MRI curves are known to be closely associated with laboratory and clinical indicators of inflammation and with histological vascularity indices, ${ }^{37}{ }^{38}$ and a very good correlation between dynamic MRI and power Doppler ultrasonographic methods has indeed been reported. ${ }^{39}$

Validation of synovial power and colour Doppler has already been assessed in several studies. ${ }^{134041}$ Computed and subjective synovial power Doppler ultrasonographic scores were recently compared with histopathological vessel scores, ${ }^{42}$ and also with arthroscopic vessel scores, ${ }^{24}$ suggesting the reliability of both unenhanced and contrast enhanced power Doppler ultrasonographic techniques.

Some limitations of our study should be considered. The accuracy of power Doppler synovial flow assessment by the latest ultrasound equipment must to be compared with contrast enhanced ultrasonographic methods. ${ }^{14} 24$ Subjective power Doppler scoring of synovial vascularity, using the semiquantitative grading scale, requires further validation by longitudinal studies of larger numbers of KJS patients and in earlier phases of rheumatoid and psoriatic KJS.

Good interobserver and intraobserver reproducibility in the ultrasonographic detection of synovitis in the knee has already been reported. ${ }^{43}$ Our findings and recent evidence ${ }^{31} 32$ further suggest that measurement of synovial thickness is an adequate method of quantifying synovitis in longitudinal studies. Using standardised anatomical guidelines, the combined grey scale and power Doppler ultrasonographic method has indeed shown itself to be sensitive in detecting time dependent rheumatoid and psoriatic KJS synovial changes in individual joints in response to therapy, paralleling changes in disease activity (fig 1).

CE-MRI is now considered to be the most valuable method for monitoring synovitis. ${ }^{9}$ Ultrasonography allows less comprehensive anatomical coverage within individual joints, but has the advantages of higher resolution of soft tissue architecture. It is also readily available, costs less, and may be undertaken by rheumatologists.

Based on clinical findings and objective ultrasonographic imaging, the addition of the anti-TNF $\alpha$ agent etanercept to the treatment regimen results in effective suppression of synovitis in rheumatoid and psoriatic knee joints that are only partially responsive to methotrexate and DMARD combinations. The similar degree of ultrasonographic synovial thickening and power Doppler vascularity changes detected in rheumatoid and psoriatic KJS after treatment 
(table 3) suggests non-disease-specific variations in knee joint synovitis. ${ }^{44}$ Nevertheless, our study does not show that the clinical and ultrasound results are unique to etanercept and it would be worthwhile evaluating what happens to KJS patients treated with other drugs using this technique. ${ }^{16} 1745$

Although the relation between synovitis and joint damage remains controversial, ${ }^{38}$ recent MRI studies have shown that effective suppression of synovitis can reverse structural damage and that there is a threshold level of synovitis for the progression of bony damage. ${ }^{46}$

Previous pilot ultrasonographic imaging studies and our own results are encouraging, and should stimulate further interest in the use of combined grey scale and power Doppler ultrasonography as an outcome measure for the response of rheumatoid and psoriatic synovitis to treatment. ${ }^{13} 47$ Ultrasonographic changes have been shown to be complementary to the standard clinical evaluation in multiple joint assessment $^{31}$ and to provide similar profiles of local and systemic disease activity over time in longitudinal monitoring of single joint response to treatment.

\section{Authors' affiliations}

M Vezzù, L Cozzi, C Checchetto, P Sfriso, C Botsios, L Ciprian, A Piccoli, $S$ Todesco, Division of Rheumatology, Department of Medical and Surgical Sciences, University of Padua, Padua, Italy

U Fiocco, G Armellin, R Nardacchione, Multidisciplinary Day Surgery Department, Padua General Hospital

F Ferro, L Rubaltelli, Department of Medical Diagnostic Sciences and Special Therapies, University of Padua

\section{REFERENCES}

1 Paget S. Recurrent synovitis of the knee in rheumatoid arthritis. In: Klippel JH, Dieppe PA, eds. Rheumatology, 2nd ed. London: CV Mosby, 1998:16.16-16.17

2 Massardo L, Gabriel SE, Crowson CS, O'Fallon WM, Matteson EL. A population based assessment of the use of orthopedic surgery in patients with rheumatoid arthritis. J Rheumatol 2002;29:52-6.

3 Sokoll KB, Helliwell PS. Comparison of disability and quality of life in rheumatoid and psoriatic arthritis. J Rheumatol 2001;28:1842-6.

4 Rahman P, Nguyen E, Cheung C, Schentag CT, Gladman DD. Comparison of radiological severity in psoriatic arthritis and rheumatoid arthritis. J Rheumatol 2001;28:1041-4.

5 Feletar M, Brockbank JE, Schentag CT, Lapp V, Gladman DD. Treatment of refractory psoriatic arthritis with infliximab: a 12 month observational study of 16 patients. Ann Rheum Dis 2001:60:233-6.

6 Yazici Y, Erkan D, Lockshin MD. A preliminary study of etanercept in the treatment of severe, resistant psoriatic arthritis. Clin Exp Rheumatol 2000; 18:732-4.

7 Marguerie L, Flipo RM, Grardel B, Beaurian D, Duquesnoy B, Delcambre B. Use of disease-modifying antirheumatic drugs in patients with psoriatic arthritis. Joint Bone Spine 2002;69:275-81.

8 Gladman DD. Effectiveness of psoriatic arthritis therapies. Semin Arthritis Rheum 2003;33:29-37.

9 Peterfy CG. New developments in imaging in rheumatoid arthritis. Curr Opin Rheumatol 2003;15:288-95.

10 Emery P. Evidence supporting the benefit of early intervention in rheumatoid arthritis. J Rheumatol Suppl 2002;66:3-8.

11 Rubaltelli L, Fiocco U, Cozzi L, Baldovin M, Rigon C, Bortoletto $P$, et al. Prospective sonographic and arthroscopic evaluation of proliferative knee joint synovitis. J Ultrasound Med 1994; 13:855-62.

12 Fiocco U, Cozzi L, Rubaltelli L, Rigon C, De Candia A, Tregnaghi A, et al. Long-term sonographic follow-up of rheumatoid and psoriatic proliferative knee joint synovitis. Br J Rheumatol 1996;35:155-63.

13 Wakefield RJ, Brown AK, O'Connor PJ, Emery P. Power Doppler sonography: improving disease activity assessment in inflammatory musculoskeletal disease. Arthritis Rheum 2003;48:285-8.

14 Klauser A, Frauscher F, Schirmer M, Halpern E, Pallwein L, Herold M, et al. The value of contrast-enhanced color Doppler ultrasound in the detection of vascularization of finger joints in patients with rheumatoid arthritis. Arthritis Rheum 2002;46:647-53.

15 Hau M, Schultz H, Tony HP, Keberle M, Jahns R, Haeten R, et al. Evaluation of pannus and vascularisation of the metacarpophalangeal and proximal interphalangeal joints in rheumatoid arthritis by high-resolution ultrasound (multidimensional linear array). Arthritis Rheum 1999;42:2303-8.

16 Newman JS, Laing TJ, McCarthy CJ, Adler RS. Power Doppler sonography of synovitis: assessment of therapeutic response - preliminary observations. Radiology 1996;198:582-4.

17 Stone M, Bergin D, Whelan B, Maher M, Murray J, McCarthy C. Power Doppler ultrasound assessment of rheumatoid hand synovitis. J Rheumatol 2001;28:1979-82.
18 Arnett FC, Edworthy SM, Bloch DA, McShane DJ, Fries JF Cooper NS, et al. The American Association 1987 revised criteria for the classification of rheumatoid arthritis. Arthritis Rheum 1988;31:315-24.

19 Moll JM, Wright V. Psoriatic arthritis. Semin Arthritis Rheum 1973:3:55-78.

20 Helliwell PS, Hethen J, Sokoll K, Green M, Marchesoni A, Lubrano E, et al Joint symmetry in early and late rheumatoid and psoriatic arthritis: comparison with a mathematical model. Arthritis Rheum 2000:43:865-71.

21 McGonagle D, Gibbon W, O'Connor P, Green M, Pease C, Emery P. Characteristic magnetic resonance imaging entheseal changes of knee synovitis in spondyloarthropathy. Arthritis Rheum 1998;41:694-700.

22 Fiocco U, Cozzi L, Rigon C, Chieco-Bianchi F, Baldovin M, Cassisi GA, et al. Arthroscopic synovectomy in rheumatoid and psoriatic knee joint synovitis: long-term outcome. Br J Rheumatol 1996;35:463-70.

23 Fiocco U, Cozzi L. Chieco Bianchi F, Rigon C, Vezzu M, Favero E, et al. Vascular changes in psoriatic knee joint synovitis. J Rheumatol 2001;28:2480-6.

24 Fiocco U, Ferro F, Cozzi L, Vezzu M, Sfriso P, Checchetto C, et al. Contrast medium in power Doppler ultrasound for assessment of synovial vascularity: comparison with arthroscopy. J Rheumatol 2003;30:2170-6.

25 Marks GC, Habicht JP, Mueller WH. Reliability, dependability, and precision of anthropometric measurements: the Second National Health and Nutrition Examination Survay, 1976-1980. Am J Epidemiol 1989;130:578-87.

26 Mease PJ, Goffe BS, Metz J, VanderStoep A, Finck B, Burge DJ. Etanercept in the treatment of psoriatic arthritis and psoriasis: a randomised trial. Lancet 2000;356:385-90.

27 Antoni C, Dechant C, Hanns-Martin Lorenz PD, Wendler J, Ogilvie A, Lueftl M, et al. Open-label study of infliximab treatment for psoriatic arthritis: clinical and magnetic resonance imaging measurements of reduction of inflammation. Arthritis Rheum 2002:47:506-12.

28 Weinblatt ME, Kremer JM, Bankhurst AD, Bulpitt KJ, Fleischmann RM, Fox RI, et al. A trial of etanercept, a recombinant tumor necrosis factor receptor: Fc fusion protein, in patients with rheumatoid arthritis receiving methotrexate. N Engl J Med 1999;340:253-9.

29 Bathon JM, Martin RW, Fleischmann RM, Tesser JR, Schiff MH, Keystone EC, et al. A comparison of etanercept and methotrexate in patients with early rheumatoid arthritis. N Engl J Med 2000;343:1586-93.

30 Gladmann DD, Farewell VT, Nadeau C. Clinical indicators of progression in psoriatic arthritis: multivariate relative risk model. I Rheumato 1995;22:675-9.

31 Ribbens C, Andre B, Marcelis S, Kaye O, Mathy L, Bonnet V, et al. Rheumatoid hand joint synovitis: gray-scale and power Doppler US quantifications following anti-tumor necrosis factor-alpha treatment: pilot study. Radiology 2003;229:562-9.

32 Taylor PC, Steuer A, Gruber J, Cosgrove DO, Blomley MJK, Marsters PA, et al. Comparison of ultrasonographic assessment of synovitis and joint vascularity with radiographic evaluation in a randomized, placebo-controlled study of infliximab therapy in early rheumatoid arthritis. Arthritis Rheum 2004;50:1107-16

33 Ostegaard M. Quantitative magnetic resonance imaging as marker of synovial membrane regeneration and recurrence of synovitis after arthroscopic knee joint synovectomy: a one year follow up study. Ann Rheum Dis 2001;60:233-6

34 Marzo-Hortega H, McGonagle D, O'Connor P, Emery P. Efficacy of etanercept in the treatment of the entheseal pathology in resistant spondylarthropathy: a clinical and magnetic resonance imaging study. Arthritis Rheum 2001;44:21 12-17.

35 Hau M, Kneitz C, Tony HP, Keberle M, Jahns R, Jenett M. High resolution ultrasound detects a decrease in pannus vascularization of small finger joints in patients with rheumatoid arthritis receiving treatment with soluble tumor necrosis factor alpha receptor (etanercept). Ann Rheum Dis 2002;61:55-8.

36 Terslev L, Torp-Pedersen S, Qvistgaard E, Kristoffersen H, Rogind H, Danneskiold-Samsøe B, et al. Effects of treatment with etanercept (Enbrel, TNRF:Fc) on rheumatoid arthritis evaluated by Doppler ultrasonography. Ann Rheum Dis 2003;62:178-81

37 Ostergaard M, Stoltenberg M, Lovgreen-Nielsen P, Volck B, Jensen CH, Lorenzen I. Magnetic resonance imaging-determined synovial membrane and joint effusion volumes in rheumatoid arthritis and osteoarthritis: comparison with the macroscopic and microscopic appearance of the synovium. Arthritis Rheum 1997;40: 1856-67.

38 Ostergaard $M$, Hansen $M$, Stoltenberg M, Gideon P, Klarlund M, Jensen KE, et al. Magnetic resonance imaging-determined synovial membrane volume as a marker of disease activity and a predictor of progressive joint destruction in the wrist of patients with rheumatoid arthritis. Arthritis Rheum 1999;42:918-29.

39 Szkudlarek M, Court-Payen M, Strandberg C, Klarlund M, Klausen T, Ostergaard M. Power Doppler ultrasonography for assessment of synovitis in the metacarpophalangeal joints of patients with rheumatoid arthritis: a comparison with dynamic magnetic resonance imaging. Arthritis Rheum 2001;44:2018-23.

40 Carotti M, Salaffi F, Manganelli P, Salera D, Simonetti B, Grassi W. Power Doppler sonography in the assessment of synovial tissue of the knee joint in rheumatoid arthritis: a preliminary experience. Ann Rheum Dis 2002;61:877-82

41 Weidekamm C, Koller M, Weber M, Kainberger F. Diagnostic value of highresolution $\mathrm{B}$-mode and Doppler sonography for imaging of hand finger joints in rheumatoid arthritis. Arthritis Rheum 2003;48:325-33.

42 Walther M, Harms H, Krenn V, Radke S, Faehndrich TP, Gohlke F. Correlation of power Doppler sonography with vascularity of the synovial tissue of the 
knee joint in patients with osteoarthritis and rheumatoid arthritis. Arthritis Rheum 2001;44:331-8.

43 Karim Z, Wakefield RJ, Quinn M, Conaghan PG, Brown AK, Veale DJ, et al. Validation and reproducibility of ultrasonography in the detection of synovitis in the knee: A comparison with arthroscopy and clinical examination. Arthritis Rheum 2004;50:387-94.

44 Rhodes LA, Tan AL, Tanner SF, Radjenovic A, Hensor EMA, Reece R, et al. Regional Variation and differential response to therapy for knee synovitis adjacent to the cartilage-pannus junction and suprapatellar pouch in inflammatory arthritis. Arthritis Rheum 2004;50:2428-32.
45 Filippucci E, Farina A, Carotti M, Salaffi F, Grassi W. Grey scale and power Doppler sonographic changes induced by intra-articular steroid injection treatment. Ann Rheum Dis 2004;63:740-43.

46 Conaghan PG, O'Connor P, McGonagle D, Astin P, Wakefield R Gibbon WW, et al. Elucidation of the relationship between synovitis and bone damage: a randomized magnetic resonance imaging study of individual joints in patients with early rheumatoid arthritis. Arthritis Rheum 2003;48:64-71.

47 Gladmann DD, Helliwell PH, Mease PJ, Nash P, Ritchlin C, Taylor W. Assessment of patients with psoriatic arthritis. A review of currently available measures. Arthritis Rheum 2004;50:24-35.

\section{$\mathrm{ECHO}$}

\section{IFN $\alpha$ combats Siögren's syndrome and its neuropathies}

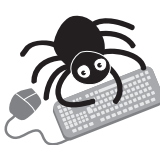

Please visit the Annals of the Rheumatic Diseases website [www. annrheumdis. com] for a link to the full text of this article. nterferon alpha (IFN $\alpha$ ) could become the preferred treatment for Sjögren's syndrome (SS), with the finding from a small case study that it alleviates not only associated neuropathic symptoms but also the syndrome itself.

This is the first report of its effect against two progressive and severely disabling neuropathies associated with the syndrome, and it will be important to assess whether this effect holds true across the full range.

IFN $\alpha$ ( $3 \mathrm{MIU} /$ day, three times a week) improved neurological symptoms and function and enabled patients to regain mobility and resume activities of daily living within one to two months after it was first given. Sicca symptoms and histological abnormalities of the salivary glands also resolved, and serum SS-A/SS-B antibodies returned to normal titres. Before receiving IFN $\alpha$ the patients had been treated variously with prednisolone, cyclophosphamide, and cyclosporine, to no avail, and with intravenous immunoglobulin, which required repeat treatments at two to three weeks to two to three months afterwards to maintain their effect.

The three cases were a 46 year old man and a 67 year old woman with sensory ataxic ganglionopathy and a 45 year old woman with demyelinating polyradiculoneuropathy. All had sufficient features to be classed as having SS, including raised titres of SS-A/SS-B antibodies and histologically abnormal salivary glands.

IFN $\alpha$ has been reported to reduce sicca symptoms, but not neuropathy, associated with SS, even though peripheral neuropathy is the commonest symptom other than abnormal functioning of the tear and salivary glands.

\ Yamada S, et al. Journal of Neurology, Neurosurgery, and Psychiatry 2005;76:576-578. 